\title{
Adrenal gland dysfunction and myocardial Tissue Doppler echocardiographic abnormalities in Patients with Beta Thalassemia Major: cross sectional study
}

\author{
Najat Mahdi $^{1,2}$, Taysir S. Garadah ${ }^{1,2,3}$, Zuheir Hassan ${ }^{4}$, Ahmed Abu-Taleb ${ }^{2}$, Das S Nagalla ${ }^{1,2}$ \\ ${ }^{1}$ Cardiac Unit, Salmaniya Medical Complex, Ministry of Health, Kingdom of Bahrain. \\ ${ }^{2}$ Department of Family and Community Medicine, ${ }^{4}$ Department of physiology, College of Medicine and Medical Sciences, Arabian \\ Gulf University, Kingdom of Bahrain. \\ ${ }^{3}$ King Hamad University Hospital, Kingdom of Bahrain. \\ *Corresponding author E-mail: garadaht@hotmail.com
}

\begin{abstract}
Background: Adrenal, parathyroid gland and myocardial echocardiographic abnormalities are recognized with variable outcomes in patients with transfusion dependent Beta-Thalassemia major (B-TM).

Aim: In this cross-sectional analytic study in patients with $\beta$-TM, we evaluate the left ventricle systolic and diastolic function using Pulsed Doppler (PD) and Tissue Doppler (DT) echocardiogram with simultaneous measurement of serum level of cortisol and parathyroid hormone.

Methods: The study was conducted on patients with $\beta$-TM $(n=99$, age $15.92 \pm 8.92$ years $)$ and compared with an agematched controls ( $\mathrm{n}=98$ age 15.79 \pm 8.94 years). In all participants echocardiographic indices of $\mathrm{M}$ mode and PD and TD were performed. Blood samples were withdrawn for measuring the serum cortisol, parathyroid and Ferritin. Correlation between the level of cortisol and ferritin level was evaluated.

Results: Patients with $\beta$-TM compared with controls, have significantly thicker LV septal wall index of $0.65 \pm 0.26 v s$. $0.44 \pm 0.2190, \mathrm{P}<0.001$ and $\mathrm{LV}$ posterior wall of $0.65 \pm 0.235$ vs. $0.43 \pm 0.214, \mathrm{P}<0.001$, with no significant dilation of

LV cavity in systole and diastole. The systolic function of LVFE\% was normal of 51.95 5.5 vs. 5.0干5.6, $\mathrm{p}=0.23$. Furthermore patients with $\beta$-TM have higher $\mathrm{E} / \mathrm{A}$ ratio $(1.54 \pm 0.18$ vs. $1.23 \pm 0.17, \mathrm{P}<0.01)$ and shorter deceleration time (DT) $(170.53 \pm 13.3 v s .210 .50 \pm 19.20 \mathrm{~m} \mathrm{sec}, \mathrm{P}<0.01)$. The ratio of transmitral $\mathrm{E}$ wave velocity to the tissue Doppler $\mathrm{E}$ wave at the basal septal mitral annulus $(\mathrm{E} / \mathrm{Em})$ was significantly higher in $\beta$-TM group (19.68 $2.81 \mathrm{vs}$. 13.86 \pm 1.41 , $\mathrm{P}<0.05)$. The tissue Doppler systolic wave $(\mathrm{Sm})$ velocity and the early diastolic wave $(\mathrm{Em})$ were significantly lower in $\beta$-TM group compared with controls with $\mathrm{Sm}$, of $4.82 \pm 1.2$ vs. $6.22 \pm 2.1 \mathrm{~cm} / \mathrm{sec}, \mathrm{P}<0.05$ and $(\mathrm{Em})$ of $3.51 \pm 2.7$ vs. $4.12 \pm 2.5 \mathrm{~cm} / \mathrm{sec} \mathrm{P}<0.05$, respectively). The tricuspid valve velocity was significantly higher in $\beta$-TM patients compared with controls $(2.85 \pm 0.56$ vs. $1.743 \pm 0.47 \mathrm{~m} / \mathrm{sec}$, respectively, $\mathrm{P}<0.01)$. The prevalence of adrenal insufficiency in patients with B-TM was $16 \%$, hypoparathyroidism of $4.5 \%$. There was a weak negative correlation between serum level of cortisol and the serum Ferritin.

Conclusion: Patients with $\beta$-thalassemia major had a high prevalence of subclinical adrenal insufficiency of $16 \%$, hypoparathyroidism of $4.5 \%$ with weak negative correlation between the low level of cortisol $\leq 160 \mathrm{nmol} / \mathrm{L}$ and high serum ferritin. Echocardiographic Pulsed Doppler showed a restrictive LV diastolic pattern suggestive of advanced diastolic dysfunction but preserved left ventricle systolic function.
\end{abstract}

Keywords: Cortisol hormone, Beta-Thalassemia Major, Pulsed echo Doppler, Tissue Doppler Echocardiography, Bahrain.

\section{Introduction}

Adrenal gland dysfunction is an uncommon endocrine complication in patients with blood transfusion dependent BetaThalassemia Major ( $\beta$-TM) [1] In patient with $\beta$-TM, repeated blood transfusion and hemolysis of red blood cell, end up with iron overload.[2] Iron deposition affects adversely the function and the structure of myocardium, liver, parathyroid, thyroid and adrenal gland. [3]

Iron chelating therapy at present is considered the standard care of this blood disorder with significant improvement of morbidity and increased rate of survival.[4] Patients with B-TM in the second decade of life and due to the lack of tolerance of chelating therapy and the repeated blood transfusion had delayed puberty, short stature and small weight 
compared with the normal people due to endocrine dysfunction namely hypogonadism, hypoadrenalism and hypothyroid dysfunction.[5]

Left Ventricle (LV) systolic and diastolic function assessed by echocardiogram in patients with B-TM showed variable abnormalities depending on the patient's disease severity and age .[6,7]

LV diastolic function had been classified into three patterns of diastolic velocities, abnormal relaxation, pseudonormal and restrictive velocity pattern depending on ratio of LV early diastolic velocity ( $\mathrm{E}$ wave) and the late filling (A wave) in addition to deceleration time of $E$ wave [8]

The LV diastolic velocity pattern depends on the degree of predominance of the altered wall stiffness or the abnormal active relaxation. The restrictive pattern is characterized by high E wave velocity, diminished A wave, short DT and very high $\mathrm{E} / \mathrm{A}$ ration $>1.8$. [9]

The Tissue Doppler echocardiography (TD) can detect regional myocardial diastolic dysfunction even in early phases of cardiac injury.[10] The TD velocity at the basal septum of mitral annulus, has been evaluated as a marker of myocardial stiffness and it was suggested that the ratio of early diastolic filling wave of PD to mitral annulus DT velocity (E/Em) does correlate positively with the LV end-diastolic pressure (LVEDP). [12] Furthermore, it was shown that TD velocities of the early diastolic wave $(\mathrm{Em})$ and systolic wave $(\mathrm{Sm})$ were both reduced in stiff hypertensive myocardium. [13]

Previous studies with multiple blood transfusions in B-TM patients showed variable prevalence of adrenal insufficiency in the range of $0-35 \%$ depending on the age of the study population, duration of blood transfusions, the level of serum Ferritin and the dose of iron chelating agent. [13, 14]

The aim in this study: (1) determine the incidence of adrenal insufficiency in patient with $\beta$-TM, (2) assess the correlation between the serum levels of cortisol hormone and serum Ferritin and (3) evaluate the echocardiographic indices of LV systolic and diastolic functions using $\mathrm{M}$ mode and pulsed Doppler in transfusion dependent $\beta$-TM patients.

\section{Material and methods}

The study subjects were 99 patients with transfusion-dependent $\beta$-TM and 98 healthy individuals as a control group. The study was conducted over 12 month period from December 2011 to December 2012. Patients selection was Consecutive from those on regular follow-up in the Pediatric Hematology clinic at Salmaniya Medical Complex (SMC) . Patients in the control group were selected from patients who were referred for assessment of Cardiac murmur in pediatric clinic in the hospital. A constitutional ethical approval was obtained for the study.

Inclusion criteria

In this cross sectional prospective study, each patient with B-TM after signing a consent form gave a blood sample and booked for an echocardiogram in the same week. The blood test was done at the end of the week just prior to the blood transfusion. Patients were excluded if they had severe liver disease, an end-stage renal disease with creatinine clearance $<30 \%$ of normal, advanced heart failure or hypertension cardiomyopathy.

\subsection{Clinical and biochemical variables}

Each patient in the study had a clinical and hematological data file including duration of disease, general examination for the pulse, blood pressure, presence or absence of pubic and axillary hair, cardiovascular assessment for apex beat displacement, jugular venous pressure wave, cardiac murmurs, lung field, hepatosplenomegaly and ankle edema.

Height and weight were obtained; the body surface area (BSA) and body mass index (BMI) were derived. The blood level of hemoglobin, serum ferritin, morning cortisol hormone, parathormone, calcium, phosphate, potassium, sodium and creatinine / estimated glomerular filtration rate (eGFR) were all recorded. The corrected calcium level was obtained by upward of calcium by $0.1 \mathrm{mmol} / \mathrm{L}$ for each $5 \mathrm{gm}$ reduction of albumin below $40 \mathrm{gm} / \mathrm{L}$.

Parathyroid hormone and cortisol were both analyzed using Chemiluminescence immune assay method with normal range of morning cortisol of (190-690 nmol/L) and parathormone of (1.0-6.5 nmol/L)

\subsection{Echocardiography}

Each patient in the study had echo examination by $2.5-5 \mathrm{MHz}$ transducer, using HP E33 echo machine. The echocardiography tests were performed by an echo technologist, who was blinded about the clinical condition of the patient. Data was reported as an average of at least five cardiac cycles. Another echo technician analyzed the data blindly and the data was taken as an average of the two readings. All measurements were conducted according to the recommendations of the American Society of Echocardiography (ASE). [15] 
Each patient had echocardiographic measurements including M-mode, 2D echo, LV diastolic filling velocities. The Mmode echo parameters including the LV septal wall thickness, posterior LV wall thickness, mid LV cavity dimension in systole (LVESD) and diastole (LVEDD)

LV fractional shortening (FS \%) and LV ejection fraction percentage (LVEF \%) were measured using Teichholz formula: $V=\left(7.0 /(2.4+D) * D^{3}\right) .(16)$ Pulsed Doppler was acquired in the apical view, while patients in the left lateral decubitus position at the end of expiration.

The tissue Doppler velocity of the basal septal mitral annulus, early filling E wave velocity (Em), the calculated E/Em ratio and the systolic wave $(\mathrm{Sm})$ were all recorded as a mean of five readings. The tricuspid valve velocity in systole $(\mathrm{cm} / \mathrm{s})$ was measured in apical view.

\subsection{Statistical analysis}

The data was entered and analyzed using the Statistical Package of Social Sciences (SPSS) version 17.0 and presented as mean \pm SD. Unpaired student $t$-test was used to analyze the differences between the variables in the control and the $\beta$-TM groups. The M-mode dimensions of the LV were adjusted and indexed for BSA of each patient. Correlation was tested between the serum level of cortisol as dependent variable and ferritin as independent variable. All other pulsed and tissue Doppler indices for systolic and diastolic functions were corrected for heart rate such as: E wave velocity, A wave velocity, DT, E/A ratio, E/Em ration, $\mathrm{S}$ wave and Em wave. The differences between groups was considered statistically significant at a probability value of $<0.05$.

\section{Results}

The demographic and biochemical data of all $\beta$-TM patients and the healthy controls are summarized in table 1 . The $\beta$ TM patients are matching with the control group in age and gender with higher preponderance of male in both groups. ß-TM patients had significantly lower body surface area and body mass index compared with control patients of $(1.16 \pm 0.11$ vs. $1.39 \pm 0.14, \mathrm{P}<0.05)$ and $(17.5 \mp 1.9$ vs. $24.16 \mp 2.7, \mathrm{P}<0.001)$ respectively. The mean difference between the serum potassium, sodium, corrected calcium and phosphate and eGFR in both groups is of no statistical significance. The serum ferritin level is significantly higher in the $\beta$-TM group compared with the control group (3337.87 \pm 741.14 $\mu \mathrm{g} / \mathrm{L} v s .220 .56 \pm 48.14 \mu \mathrm{g} / \mathrm{L}, \mathrm{P}<0.001)$.

The mean of serum cortisol and parathyroid hormones in B-TM patients are significantly lower than the control of

(251.6干63.7 vs. $484.7 \mp 84.3 \mathrm{nmol} / \mathrm{L}, \mathrm{p}<0.001$.) and (respectively $1.4 \mp 1.2$ vs. $4.5 \mp 2.7 \mathrm{nmol} / \mathrm{L}, \mathrm{P}<0.001)$. Pearson correlation test between ferritin and cortisol equals to -0.334 ( $\mathrm{p}$-value $=0.001$ ). There is a weak negative relationship between ferritin and cortisol. This means that as the ferritin level increase the cortisol level decrease.

Table 2 shows the hemodynamic and echocardiographic variables in both groups. The blood pressure in systole and diastole and the heart rate were with no significant differences. In $\beta$-TM patients compared with control, the indexed M-mode measurements showed significantly thicker LV inter-ventricular septum and posterior wall, ( $\mathrm{P}<0.001$ in both). Further the LV cavity indexed dimensions at the end of systole (LVESD) and diastole (LVEDD) were within normal with no significant difference. Indexed left ventricle mass was significantly higher in $\beta$-TM group compared with the control (129.69干7.4 vs. $104.55 \pm 8.7$ respectively, $\mathrm{P}<.01)$. The LVEF\% was lower $(51.95 \mp 5.5$ vs. $53.30 \mp 5.6, \mathrm{P}<0.23)$ but with no significant difference and LV fractional shortening of percentage of $32.36 \mp 3.4 v s .33 .41 \pm 3.36 \mathrm{p}<0.12$ with no statistical difference.

Pulsed Doppler velocities of LV in diastole showed significantly higher E/A ratio in $\beta$-TM group compared with control with significantly shorter interval of DT time. The ratio of transmitral E wave velocity to TD (Em) of the septal mitral annulus $(\mathrm{E} / \mathrm{Em})$ was significantly higher in the $\beta$-TM group compared to the control group $(\mathrm{P}<0.05)$, while the early diastolic velocity $\mathrm{Em})$ and the systolic wave velocity $(\mathrm{Sm})$ were significantly lower in $\beta$-TM group $(\mathrm{P}<0.05$.). The tricuspid valve velocity was significantly higher in $\beta$-TM group at $\mathrm{P}<0.01$.

In patients with $\beta$-TM group, the color flow pattern and continuous wave velocity of the LV inflow and outflow showed mild mitral regurgitation in eight patients and three patients had mild aortic regurgitation with none in the control group. There was weak negative correlation between the serum level cortisol and serum ferritin in patient with B-TM.

Table 3 summarizes the mean value of biochemical and echocardiographic variables in $B$-TM patients with low serum cortisol level $\leq 190 \mathrm{nmol} / \mathrm{L}(\mathrm{n}=16)$ and those with $1>190 \mathrm{nmol} / \mathrm{L}(\mathrm{n}=83)$.

Patients with serum cortisol $>190 \mathrm{nmol} / \mathrm{L}$ in comparison with patients with low cortisol $\leq 190 \mathrm{nmol} / \mathrm{L}$ had significantly higher ferritin level >3500 ng/l, a lower value of eGFR, higher LV mass and higher E/A ratio on pulsed Doppler. 


\section{Discussion}

Ninety nine patients with $\beta$-TM were evaluated in this study. They were evaluated for presence or absence of adrenal and parathyroid dysfunction clinically and biochemically in addition to pulse Doppler echocardiogram for systolic and diastolic function of left ventricle.

It was reported that the rate of incidence of adrenal gland dysfunction in B-TM patients is of lower incidences relative to other glands such as thyroid and parathyroid. [5] In one report of one hundred and fifty patients with B-TM the incidence of hypoparathyroidism was $7.5 \%$, hypothyroidism of $6 \%$, DM of $7.3 \%$ and only one patient had adrenal insufficiency $(0.06 \%)$. (1) Elsedfy et al., reported adrenal insufficiency in 7 of 45 (15\%) patients with $\beta$-TM. [13]

Bashir et al., (Jordan) reported a low cortisol level in $57 \%$ of patients with thalassemia with mean cortisol level of $182 \mp 32 \mathrm{nmol} / \mathrm{L}$ compared with $420 \mp 100 \mathrm{nmol} / \mathrm{L}$ in control group indicating in vivo hypoadrenalism. [17]

In one study of 435 there were $32.5 \%$ patients with hypogonadotrophic hypogonadism and reduced pubertal growth spurt stature in 35\%, acquired hypothyroidism in 5.9\%, hypoparathyroidism in $1.2 \%$ and DM in 9.4\%. [5]

Delayed puberty and short stature of adrenal insufficiency are common findings but it seems adrenal pituitary axis is almost not affected. However the morning cortisol level of $<400 \mathrm{nmol} / \mathrm{L}$ was accepted as an indicator of adrenal insufficiency. [18]

So it seems the variation in adrenal dysfunction among different reports depend on the age of patient at the time of blood assay, the active use of chelating and duration of blood transfusion therapy.

The variation of prevalence in the past may be related to different methods used for adrenal function studies, different ages in the study or different medications.

The data in this study showed poor correlation between serum iron and serum cortisol which is in keeping with other reports where mean cortisol level was significantly low in comparison with control, with negative correlation between serum level of ferritin and cortisol. [19]

In this study, the incidence of in vitro low cortisol level $<190 \mathrm{nmol} / \mathrm{L}$ was detected in $16 \%$, hypoparathyroidism recorded in $4.5 \%$ with $34 \%$ of patients below the fifth percentile of height, with no delay of appearance of pubic and auxiliary hair.

The diastolic indices of LV in $\beta$-TM patients showed significantly higher early diastolic filling of LV and E/A ratio suggesting restrictive diastolic pattern and stiff myocardial wall.

These findings are in keeping with one study by Yaprak et al.,[20] who demonstrated that $\beta$-TM patients had significantly higher $\mathrm{E}$ wave, E/A ratio, and lower A wave velocity, suggesting a restrictive pattern but with no correlation with hemoglobin level. Similar observations were reported by Spirito et al., who demonstrated a restrictive pattern in patients with $\beta$-TM with no heart failure. This was also in agreement with a previous report that high E/A ratio is the most common finding in patients with $\beta$-TM [21].

The decreased DT of E wave was mostly related to the increased amplitude of the $\mathrm{E}$ wave and may be due to the impaired relaxation of LV with a constrictive pattern. The exact mechanism of these findings is not known but may be due to iron overload and increased stiffness of LV wall.

The tissue Doppler ratio of E/Em was significantly higher in $\beta$-TM patients compared with controls. Likewise, the early diastolic velocity $(\mathrm{Em})$ and the systolic velocity wave $(\mathrm{Sm})$ of the basal septum were significantly reduced, suggesting stiff myocardium and possibly high LVEDP. These findings are in agreement with those previously reported in hypertensive patients. [22, 23]

The LVEF\% was normal indicating that $\beta$-TM patients are having a preserved systolic function. In the current study, the serum ferritin was fifteen times higher in the $\beta$-TM group compared with controls. These findings confirm our previously published data showing impaired diastolic function leading to stiffness of the myocardial wall but with wellpreserved LV systolic function. [24]

It seems in this population as observed previously, myocardial disease go through a stage of impaired relaxation before development of systolic dysfunction. This finding is in agreement with one report where patients with $\beta$-TM with no heart failure had higher E/A ratio compared with controls. [6]

\section{Conclusion}

We conclude that patients with $\beta$-TM had a high prevalence of subclinical hypoadrenalism of $16 \%$, hypoparathyroidism of $4.5 \%$ with weak negative correlation between the low level of cortisol $<160 \mathrm{nmol} / \mathrm{L}$ and high serum ferritin.

Echocardiographic Pulsed Doppler showed a restrictive LV diastolic pattern suggestive of diastolic dysfunction with preserved left ventricle systolic function. 
Table 1: Demographic characteristics of control $(n=98)$ and thalassemia patients $(n=99)$ in the study. Data are presented as mean \pm SD. $P$ value of $<$ 0.05 is considered statistically significant.

\begin{tabular}{|l|l|l|l|}
\hline & Thalssemia: $\mathrm{n}=99$ & Control: $\mathrm{n}=98$ & P value \\
\hline Age in years & $14.77 \mp 3.9$ & $14.66 \mp 3.3$ & 0.82 \\
\hline Male & $58(58.5 \%)$ & $55(56 \%)$ & 0.92 \\
\hline Female & $41(41.5 \%)$ & $43(44 \%)$ & 0.45 \\
\hline BSA & $1.16 \mp 0.11$ & $1.39 \mp 0.14$ & 0.012 \\
\hline Body mass index & $17.5 \mp 1.9$ & $24.16 \mp 2.7$ & 0.001 \\
\hline Serum Cortisol nmol/L & $251.05 \pm 83.72$ & $284.78 \pm 89.39$ & 0.007 \\
\hline Serum parathyroid hormone nmol/L & $1.26 \pm 0.08$ & $4.46 \pm 0.22$ & $<0.001$ \\
\hline Serum calcium mmol/L & $2.20 \pm 0.10$ & $2.40 \pm 0.13$ & $<0.001$ \\
\hline Serum phosphate mmol/L & $1.80 \pm 0.08$ & $1.25 \pm 0.08$ & $<0.001$ \\
\hline HB gm/dl & $10.7 \mp 0.81$ & $11.1 \mp 0.91$ & 0.19 \\
\hline Serum Ferritin $\mu$ gm/L & $3337.87 \mp 741.14$ & $220.56 \mp 48.14$ & 0.001 \\
\hline Serum potassium Potassium mmol/L & $3.95 \mp 0.45$ & $3.91 \mp 0.44$ & 0.86 \\
\hline Serum Sodium mmol/L & $137.49 \mp 3.4$ & $135.41 \mp 3.4$ & 0.48 \\
\hline eGFR & $77.31 \pm 11.14$ & $79.05 \pm 8.40$ & 0.023 \\
\hline
\end{tabular}

Abbreviations: BSA: body surface area; eGFR: estimated Glomerular Filtration Rate.

Table 2: The hemodynamic and echocardiographic indexed M-Mode and Pulsed and tissue Doppler indices in $\beta$-thalassemia ( $\beta$-TM) patients and the control group.

Data are presented as mean \pm SM. $\mathrm{P}<0.05$ is considered statistically significant

\begin{tabular}{|l|l|l|l|}
\hline & Thalssemia: $\mathrm{n}=99$ & Control: $\mathrm{n}-98$ & $\mathrm{P}$ value \\
\hline Systolic blood pressure $\mathrm{mmHg}$ & $134.03 \pm 7.71$ & $133.26 \pm 7.19$ & 0.46 \\
\hline Diastolic Blood pressure $\mathrm{mmHg}$ & $73.43 \pm 5.61$ & $72.26 \pm 6.21$ & 0.30 \\
\hline Heart rate per minute & $72.82 \pm 3.40$ & $73.49 \pm 3.23$ & 0.153 \\
\hline LV IVS cm/M2 & $0.79 \pm 0.03$ & $0.53 \pm 0.02$ & 0.001 \\
\hline LV PW cm/M2 & $0.67 \pm 0.02$ & $0.44 \pm 0.01$ & 0.001 \\
\hline LV EDD cm/M2 & $4.48 \pm 0.20$ & $4.35 \pm 0.17$ & 0.001 \\
\hline LVESD cm/M2 & $3.23 \pm 0.26$ & $2.80 \pm 0.09$ & 0.001 \\
\hline LV mass gm/M2 & $140.33 \pm 5.48$ & $87.67 \pm 1.25$ & 0.001 \\
\hline LV EF\% & $51.95 \mp 5.5$ & $53.30 \mp 5.6$ & 0.23 \\
\hline LV FS\% & $31.368 \pm 4.36$ & $32.41 \pm 3.360$ & 0.107 \\
\hline E/A ratio & $1.71 \mp 0.41$ & $1.41 \mp 0.29$ & 0.001 \\
\hline DT in msec & $201.12 \mp 34.1$ & $246.23 \mp 41.2$ & 0.001 \\
\hline E/Em & $19.684 \pm 2.81$ & $13.868 \pm 1.41$ & 0.05 \\
\hline Em wave in mm/s & $3.51 \pm .2 .7$ & $4.12 \pm 2.5$ & 0.04 \\
\hline S wave in mm/s & $4.82 \pm 1.2$ & $6.22 \pm 2.1$ & 0.02 \\
\hline Tricuspid velocity cm/s & $2.853 \pm 0.569$ & $1.743 \pm 0.471$ & 0.045 \\
\hline
\end{tabular}

Abbreviations: IVS: inter ventricular septal thickness, PW: posterior wall thickness, LVEDD: left ventricle end diastolic diameter, LVESD: left ventricle end systolic diameter, LVEF: left ventricular ejection fraction. E: early diastolic flow velocity; A, late diastolic flow velocity; DT: deceleration time of flow velocity in early diastole. Sm: systolic myocardial velocities of basal segments of LV septal wall, (Em) early diastolic myocardial velocities of the same basal segments.

Table 3: Biochemical and echocardiographic data of patients with low cortisol compared to normal cortisol level in patients with TM.

\begin{tabular}{|l|l|l|l|}
\hline & Cortisol $>190 \mathrm{nmol} / \mathrm{L}: \mathrm{N}=83$ & Cortisol $\leq 190 \mathrm{nmol} / \mathrm{L}: \mathrm{N}=16$ & $\mathrm{P}$ value \\
\hline Age years & $15.6 \mp 3.1$ & $14.6 \mp 5.5$ & 0.34 \\
\hline eGFR & $71.2 \mp 6.2$ & $62.2 \mp 9.2$ & $<0.001$ \\
\hline Ferritin $\mu \mathrm{gm} / \mathrm{L}$ & $3130.61 \mp 480.05$ & $4400.00 \mp 933.81$ & $<0.001$ \\
\hline LV mass gm & $121.6 \mp 7.3$ & $128.4 \mp 6.3$ & 0.005 \\
\hline E/A ratio & $1.7 \mp 0.31$ & $2.1 \mp 0.41$ & $<0.001$ \\
\hline
\end{tabular}




\section{References}

[1] Gulati R, Bhatia V, Agarwal SS. Early onset of endocrine abnormalities in beta-thalassemia major in a developing country. Journal of pediatric endocrinology \& metabolism : JPEM.13(6)(2000) 651-6.

[2] Fujita S. Congenital hemolytic anemia--hemoglobin abnormality--thalassemia. Nihon rinsho Japanese journal of clinical medicine. 54(9) (1996)2454-9.

[3] Fosburg MT, Nathan DG. Treatment of Cooley's anemia. Blood.76(3) (1990) 435-44.

[4] Ehlers KH, Levin AR, Markenson AL, Marcus JR, Klein AA, Hilgartner MW, Engle MA. Longitudinal study of cardiac function in thalassemia major. Annals of the New York Academy of Sciences. 344(1980)397-404.

[5] Toumba M, Sergis A, Kanaris C, Skordis N. Endocrine complications in patients with Thalassaemia Major. Pediatric endocrinology reviews $: 5(2)(2007) 642-8$.

[6] Gharzuddine WS, Kazma HK, Nuwayhid IA, Bitar FF, Koussa SF, Moukarbel GV, Taher AT. Doppler characterization of left ventricular diastolic function in beta-thalassaemia major. Evidence for an early stage of impaired relaxation. European journal of echocardiography : the journal of the Working Group on Echocardiography of the European Society of Cardiology. 3(1) (2002)47-51.

[7] Nishimura RA, Schwartz RS, Tajik AJ, Holmes DR, Jr. Noninvasive measurement of rate of left ventricular relaxation by Doppler echocardiography. Validation with simultaneous cardiac catheterization. Circulation. 88(1)(1993)146-55.

[8] Choong CY, Herrmann HC, Weyman AE, Fifer MA. Preload dependence of Doppler-derived indexes of left ventricular diastolic function in humans. Journal of the American College of Cardiology. 10(4) (1987) 800-8.

[9] Aessopos A, Deftereos S, Tsironi M, Karabatsos F, Yousef J, Fragodimitri C, Hatziliami A, Karagiorga M. Predictive echo-Doppler indices of left ventricular impairment in B-thalassemic patients. Annals of hematology. 86(6) (7007):429-34.

[10] Nagueh SF, Middleton KJ, Kopelen HA, Zoghbi WA, Quinones MA. Doppler tissue imaging: a noninvasive technique for evaluation of left ventricular relaxation and estimation of filling pressures. Journal of the American College of Cardiology.30(6) (1997)1527-33.

[11] Ommen SR, Nishimura RA, Appleton CP, Miller FA, Oh JK, Redfield MM, Tajik AJ. Clinical utility of Doppler echocardiography and tissue Doppler imaging in the estimation of left ventricular filling pressures: A comparative simultaneous Doppler-catheterization study. Circulation. 102(15) (2000):1788-94.

[12] Kobayashi T, Tamano K, Takahashi M, Honda T, Uetake S, Ohrui M. [Myocardial systolic function of the left ventricle along the long axis in patients with essential hypertension: a study by pulsed tissue Doppler imaging]. Journal of cardiology.41(4 (2003):175-82.

[13] Elsedfy HH, El Kholy M, Tarif R, Hamed A, Elalfy M. Adrenal function in thalassemia major adolescents. Pediatric endocrinology reviews 8 Suppl 2 (2011)295-9.

[14] Kwan EY, Lee AC, Li AM, Tam SC, Chan CF, Lau YL, Low LC. A cross-sectional study of growth, puberty and endocrine function in patients with thalassaemia major in Hong Kong. Journal of paediatrics and child health. 31(2) (1995)83-7.

[15] Sahn DJ, DeMaria A, Kisslo J, Weyman A. Recommendations regarding quantitation in M-mode echocardiography: results of a survey of echocardiographic measurements. Circulation. 58(6) (1978)1072-83.

[16] Devereux RB, Alonso DR, Lutas EM, Gottlieb GJ, Campo E, Sachs I, Reichek N. Echocardiographic assessment of left ventricular hypertrophy: comparison to necropsy findings. The American journal of cardiology 57(6) (1986)450-8.

[17] Bashir N, al-Hader AF, al-Shareef L. Cortisol levels in children with haemoglobinopathies in north Jordan. Journal of tropical pediatrics.39(1) (1993)30-1.

[18] Srivatsa A, Marwaha RK, Muraldharan R, Trehan A. Assessment of adrenal endocrine function in Asian thalassemics. Indian pediatrics. 42(1) (2005)31-5.

[19] El-Hazmi MA, Warsy AS, al-Fawaz I. Iron-endocrine pattern in patients with beta-thalassaemia. Journal of tropical pediatrics.40(4) (1994) 219-24.

[20] aprak I, Aksit S, Ozturk C, Bakiler AR, Dorak C, Turker M. Left ventricular diastolic abnormalities in children with beta-thalassemia major: a Doppler echocardiographic study. The Turkish journal of pediatrics. 40(2) (1998)201-9.

[21] Spirito P, Lupi G, Melevendi C, Vecchio C. Restrictive diastolic abnormalities identified by Doppler echocardiography in patients with thalassemia major. Circulation.82(1) (1990)88-94.

[22] Bosi G, Crepaz R, Gamberini MR, Fortini M, Scarcia S, Bonsante E, Pitscheider W, Vaccari M. Left ventricular remodelling, and systolic and diastolic function in young adults with beta thalassaemia major: a Doppler echocardiographic assessment and correlation with haematological data. Heart.89(7)( 2003)762-6.

[23] Yu CM, Sanderson JE, Marwick TH, Oh JK. Tissue Doppler imaging a new prognosticator for cardiovascular diseases. Journal of the American College of Cardiology.49(19) (2007)1903-14.

[24] Garadah TS, Kassab S, Mahdi N, Abu-Taleb A, Jamsheer A. Pulsed and Tissue Doppler Echocardiographic Changes in Patients with Thalassemia Major. Clinical Medicine Insights: Blood Disorders 3 (2010)1-8.

[25] Oki T, Tabata T, Yamada H, Wakatsuki T, Mishiro Y, Abe M, Onose Y, Iuchi A, Ito S. Left ventricular diastolic properties of hypertensive patients measured by pulsed tissue Doppler imaging. Journal of the American Society of Echocardiography : official publication of the American Society of Echocardiography. 11(12) (1998) 1106-12. 